\title{
Analysis of thermal-force coupling stress field under the temperature of alternating of molecular sieve adsorption tower
}

\author{
Wang Chuanping ${ }^{1}$, Feng Xuezhang ${ }^{1}$, Yang Xiaoli ${ }^{1}, \mathrm{He} \mathrm{Sha}^{2}$, Li Yingjie ${ }^{1}$, Zhang Jia ${ }^{2, *}$ \\ ${ }^{1}$ The First Gas Production Plant of Xinjiang Oilfield Company, Xinjiang, Karamay, 834000, China \\ ${ }^{2}$ State Key Laboratory of Oil and Gas Reservoir Geology and Exploration, Southwest Petroleum University, Chengdu, Sichuan, \\ 610500, China
}

\begin{abstract}
The thermal stress of molecular sieve adsorption tower under transient temperature of $40-290^{\circ} \mathrm{C}$ is the basis for ensuring the safe operation of the adsorption tower. In this paper, based on the transient thermodynamics theory, the finite element model of the full-size adsorption tower is established. The distribution of thermal stress at the key positions of the tower body is analyzed, and the strength of the maximum equivalent stress position is evaluated. The results show that the maximum residual stress is at the corner of the inner wall of the tower opening to take over the import and export, the maximum is 313.34MPa, and the effect force is gradually diffused along the takeover; The thermal stress on the inside and outside of the skirt is greater than the thermal stress on the inside and outside of the head. The corresponding stress linearization results of each assessment path were evaluated and passed. The strength design, life prediction and maintenance of adsorption tower in complex temperature cross-change conditions provide theoretical basis.
\end{abstract}

\section{Introduction}

Fatigue damage of pressure vessels has become the most common form of failure in the petrochemical industry, and container damage can easily lead to the leakage of harmful media, causing serious environmental pollution and casualties. According to foreign pressure vessel failure accident statistics, fatigue failure accounts for about 30\% [1-3]. The failure is caused by the stress concentration of the vessel under alternating loads. Finally, the pressure vessel fatigue fracture failure [4].

Much research has been done on fatigue failure of pressure vessels under temperature stress loading. K. Hashimoto [5] performed a steady-state thermal stress analysis of the three reactor lower head using the finite element software ABAQUS, and the results showed that the peak pull stress was mainly distributed near the welding area of the nozzle. V. Chaudhry [6] used numerical models to fully evaluate thermal stresses under the stable state of the reactor pressure vessel (reactor start-up, shutdown, etc.). The results show that the position where the wall stress is greatest is in the interface between the shell and the container. D. Ferreño [7] numerically simulated the thermal stress process of the reactor pressure vessel, obtained the dynamic reference temperature $\mathrm{T}_{0, \mathrm{dyn}}$, corresponding to the loading rate under thermal shock, and compared it with the quasi-static reference temperature $\mathrm{T}_{0, \text { sta. }}$ A. Kandil [8] analyzed the stress distribution of cylindrical pressure vessels under the joint action of steady-state pressure and temperature, and obtained the relationship between average stress and stress amplitude under different operating conditions. Liu [9] analyzed the structural stress and fatigue of the adsorption tower model by using ANSYS software, taking into account the structure of the adsorption tower and the load on the nozzle and other factors. Wang [10] used finite meta-method to calculate the structural temperature stress at the stable temperature of the film-type LNG ship, and the results showed a good linear relationship between the temperature stress at the folding angle of the bottom compartment and the temperature difference under the waterline of the outer plate of the hull.

At present, fatigue failure analysis of pressure vessels is based on constant load, and most of them are based on linear elastic assumption. There are no relevant research reports on thermal stress distribution law of the tower under temperature alternating load. Based on the mechanism of transient thermodynamics, the coupling field of adsorption column under temperature alternating load is analyzed. The distribution law of instantaneous thermal stress of adsorption tower is revealed. It provides theoretical reference for the structural design of adsorption tower pressure vessel.

\section{Theoretical model}

Temperature difference exists in the three directions of the cylinder temperature field. In the case of heat transfer, the temperature at any point of radius $r$ on the wall is $t$, which can be obtained according to the equal heat conduction through each layer [11,12]. 


$$
t_{r}=\frac{t_{0} \ln \frac{r}{R_{i}}-t_{i} \ln \frac{r}{R_{0}}}{\ln \frac{R_{0}}{R_{i}}}
$$

$t_{0}$-temperature on the outer surface of the cylinder, $\mathrm{K}$; $t_{\mathrm{i}}$-temperature on the inner surface of the cylinder, $\mathrm{K}$;

$R_{\mathrm{o}}$-outer diameter of the cylinder, $\mathrm{mm}$;

$R_{\mathrm{i}}$-the inner diameter of the cylinder, $\mathrm{mm}$.

According to the knowledge of elasticity, when the temperature is distributed logarithmically along the wall thickness, the corresponding radial thermal stress $\sigma_{r}^{t}$, circular thermal stress $\sigma_{\theta}^{t}$ and axial thermal stress $\sigma_{\varphi}^{t}$ are respectively,

$$
\begin{aligned}
\sigma_{r}^{t} & =\frac{E \alpha \Delta t}{2(1-\mu)}\left(-\frac{\ln K_{r}}{\ln K}+\frac{K_{r}^{2}-1}{K^{2}-1}\right) \\
\sigma_{\theta}^{t} & =\frac{E \alpha \Delta t}{2(1-\mu)}\left(\frac{1-\ln K_{r}}{\ln K}+\frac{K_{r}^{2}-1}{K^{2}-1}\right) \\
\sigma_{\varphi}^{t} & =\frac{E \alpha \Delta t}{2(1-\mu)}\left(\frac{1-2 \ln K_{r}}{\ln K}-\frac{2}{K^{2}-1}\right)
\end{aligned}
$$

$\alpha$-linear expansion coefficient;

$\Delta t$-temperature difference between inner and outer wall of cylinder ;

$K_{\mathrm{r}}$-ratio of radius at any radius

\section{Design parameters and numerical model analysis}

\subsection{Design parameters}

The medium of a molecular sieve dehydration and adsorption tower is wet natural gas, the inner diameter of the tower is $2200 \mathrm{~mm}$, the design pressure is $7.8 \mathrm{MPa}$, the design temperature is $-20 / 320^{\circ} \mathrm{C}$, the working pressure is 7.1-7.2MPa, the working temperature is $40-290{ }^{\circ} \mathrm{C}$, and the design service life is 20 years [13].

\subsection{Numerical model}

According to the structural characteristics and load conditions of the adsorption tower as shown in figure 1. A full-size numerical model of the adsorption tower is established to analyze and evaluate the safety risks of the tower under temperature alternating loads. In order to reduce the amount of calculation, the $\mathrm{YZ}$ plane is the symmetrical cross section, and the $1 / 2$ symmetric model is used for calculation. The boundary conditions are consistent with the real working conditions. The operating temperature and pressure are the field operating conditions. The operating temperature ranges from $40{ }^{\circ} \mathrm{C}$ to $290^{\circ} \mathrm{C}$ and the operating pressure is 7.2MPa. Under the action of pressure and temperature inside the adsorption tower, the outer surface of the tower body is set as the adiabatic boundary condition because of the existence of insulation materials, and the heat flux is close to zero. The bottom of the model is fixed with constraints to limit the displacement of the rigid body, while the upper part is kept in a free state. The symmetry plane is symmetric with constraints, and the end of the pipe is subjected to an axial balance load. To solve the problem of thermal-mechanical coupling, the transient module is used for thermal stress analysis. Because that the head, the skirt and the pipe are the key research objects, paths $\mathrm{A} \sim \mathrm{H}$ as shown in the figure below are taken to analyze the distribution rule of thermal stress on each path. The influence of mesh sensitivity was optimized, and several simulations were carried out under different cell sizes to obtain the best mesh quality.

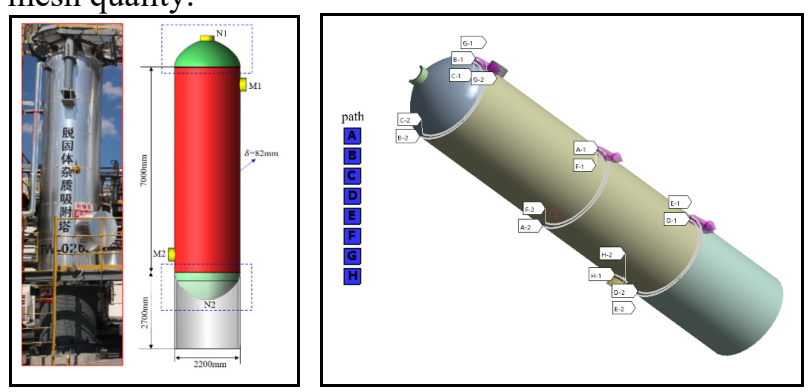

(a) Calculation model

(b) Linearized path

Fig. 1. Calculation model and linearization path.

\section{Thermal stress analysis}

To quantitatively determine whether the adsorption column tube wall damage occurs, this paper only studies the damage accumulation when the equivalent thermal stress is higher than the yield strength. Therefore, the fatigue failure criterion is simplified to judge whether damage occurs by stress concentration. The thermal stress distribution of the tower under temperature alternating load is shown in Figure 2.

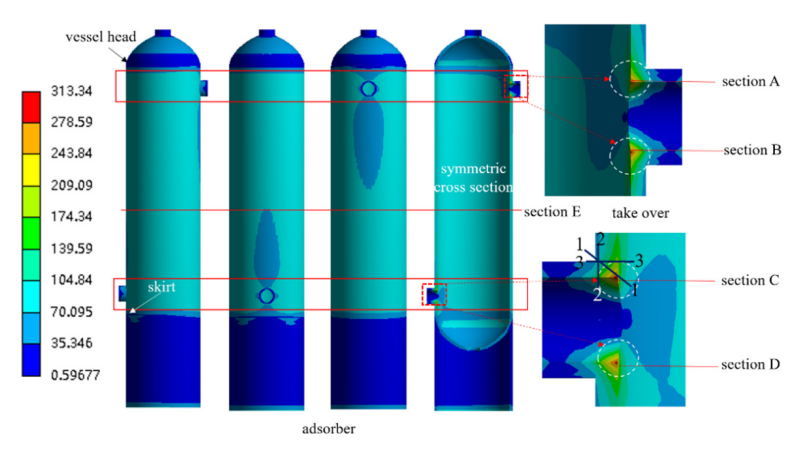

Fig. 2. Finite element analysis results.

As can be seen from Fig. 2, due to the thermal shock effect inside the tower, the horizontal temperature field keeps rising, and the superposition effect of temperature difference makes the tower body in a high stress field, and the stress value along the wall thickness of the tower gradually decreases. Under the action of alternating temperature, the equivalent stress of the adsorption column mostly concentrates between $0 \sim 104.84 \mathrm{MPa}$, which is less than the yield strength of the column body, and the plastic yield of the adsorption column does not appear. The abnormal high stress values all appear at the 
M1 and M2 openings on the side of the tower body, which is a stress singularity phenomenon. The reason for this phenomenon is that under the action of thermal stress, the tower has film stress, tower body hole reduces bearing section, makes the average stress of the cross section increases, due to the deformation coordination, take over and shell junction is a pair of shears and bending moment, thus on the edge of the hole grooving and take over the end of the local bending stress, local stress concentration makes objects produce fatigue crack.

\subsection{Stress analysis of weld joint position between skirt and tower body}

Figure 3 shows the stress distribution at the joint position of the welding seam between the skirt and the tower body. It can be seen from the figure 3 that the equivalent stress distribution of the path on the inner surface and the outer surface has a large non-uniformity. The maximum equivalent stress on the inner surface is $91.79 \mathrm{MPa}$, the maximum equivalent stress on the outer surface is $97.46 \mathrm{MPa}$, and the maximum equivalent stress is $30.1 \%$ and $32.0 \%$ of the maximum yield strength of the tower body. No plastic deformation appears in the joint position between the skirt and the tower body. Due to the wall thickness of the tower, the temperature distribution trends on the inner and outer surfaces are opposite, and the wave peak on the inner surface is the wave trough on the outer surface. In addition, the stress on the inner surface gradually decreases with the increase of time. The reason is that in the heating stage, the temperature difference of the inner wall of the tower gradually decreases under the transient temperature, the equivalent stress also decreases, while the equivalent stress on the outer surface gradually increases with the increase of the temperature difference.

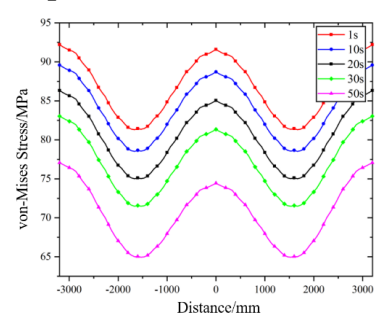

(a) Inner surface of the skirt

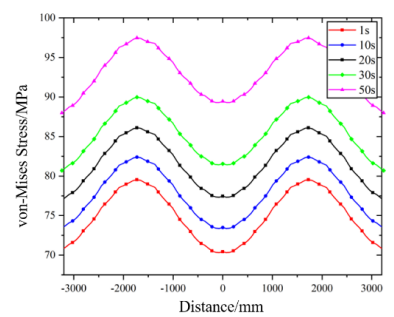

(b) Outer surface of the skirt
Fig.3. Stress distribution law of joint position between skirt and tower body.

\subsection{Stress analysis of welding joint position between head and tower body}

The circular path of the welding seam between the head and shell is taken to calculate and analyze the stress, and the calculated results are displayed symmetrically to obtain the distribution law of thermal stress at different times.

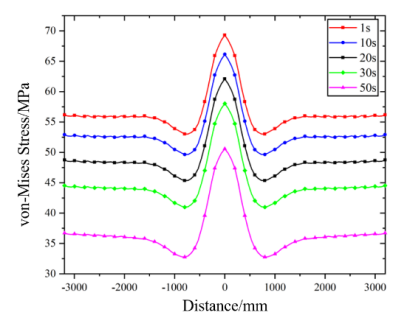

(a) Inside surface of head

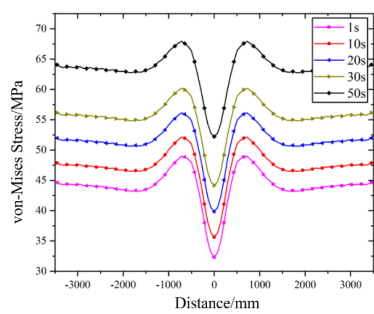

(b) Outer surface of the head
Fig.4. Stress distribution law of joint position between head and shell.

Figure 4 shows the stress distribution at the junction position of the welding seam between the head and the shell. It can be seen that the stress on the path of the inner surface and the outer surface had a certain influence at different times. The influence range of the head on the strength of the tower body is small. The stress on the path of the inner and outer surface only changes sharply near the opening position, the maximum stress is $69.32 \mathrm{MPa}$ and $67.95 \mathrm{MPa}$, the maximum equivalent stress is $22.73 \%$ and $22.28 \%$ of the maximum yield strength of the tower body, and no plastic deformation occurs. With the increase of calculation time, the temperature difference of inner surface decreases gradually, and the equivalent stress decreases. Compared with the inner surface, the temperature difference on the outer surface is larger than that on the inner surface, so the stress on the outer surface gradually increases. The effect of stress distribution on the inner surface is greater than that on the outer surface.

\subsection{Stress distribution at the nozzle}

Due to the maximum stress concentration is located at the open-hole nozzles of the tower body M1 and M2, so this section analyzes the stress distribution at the openhole nozzles of the tower body. The arcs at the open-hole nozzles of M1 and M2 are taken as paths, and the arcs on the inner surface are selected. The calculation results are shown in Figure 5.

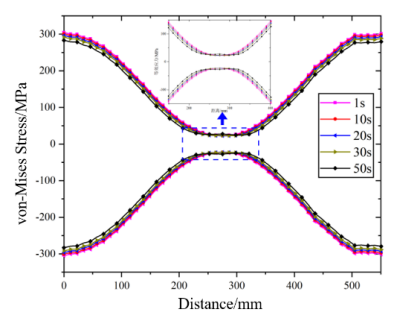

(a) Inner surface of the M1

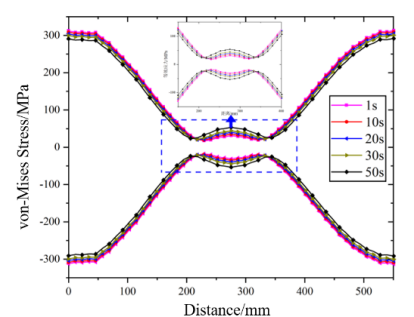

(b) Inner surface of the M2
Fig.5. Stress distribution on the inner surface of the M1and M2 nozzles.

As can be seen from Fig. 5, the circular arc length path of the nozzle is $550 \mathrm{~mm}$, and the stress on the inner and outer surfaces has basically the same trend, showing a " $U$ " shaped distribution. The maximum stress reaches 313.34MPa and $-313.22 \mathrm{MPa}$ (negative $\mathrm{Y}$ axis indicates the opposite direction), and the stress concentration coefficient is 2.54 and 2.55 respectively. With the 
prolongation of time, the equivalent stress decreases gradually, but the decreasing trend is relatively slow.

The stress linearization path was set along the shortest direction of the wall thickness along the maximum stress intensity point (M2) for strength assessment. The linearization path is shown in figure 2, which are paths 1-1, 2-2 and 3-3 respectively. The path of strength assessment results such as table 1 . The corresponding stress linearization results of each assessment path passed the assessment, and the strength assessment was all qualified.

Table 1. Assessment table of stress intensity for upper path of M2 pipe.

\begin{tabular}{ccccc}
\hline $\begin{array}{c}\text { Stress } \\
\text { strength }\end{array}$ & $\begin{array}{c}\text { Stress } \\
\text { intensity/MPa }\end{array}$ & $\begin{array}{c}\text { Stress } \\
\text { intensity/MPa }\end{array}$ & $\begin{array}{c}\text { Evaluation } \\
\text { result }\end{array}$ & Path \\
\hline $\mathrm{S}_{\mathrm{II}}$ & 145.2 & $1.5 \mathrm{~S}_{\mathrm{m}}=184.5$ & Pass & $1-1$ \\
$\mathrm{~S}_{\mathrm{IV}}$ & 227.8 & $3 \mathrm{~S}_{\mathrm{m}}=369$ & Pass & \\
$\mathrm{S}_{\mathrm{II}}$ & 138.2 & $1.5 \mathrm{~S}_{\mathrm{m}}=184.5$ & Pass & $2-2$ \\
$\mathrm{~S}_{\mathrm{IV}}$ & 189.3 & $3 \mathrm{~S}_{\mathrm{m}}=369$ & Pass & \\
$\mathrm{S}_{\mathrm{II}}$ & 114.6 & $1.5 \mathrm{~S}_{\mathrm{m}}=184.5$ & Pass & $3-3$ \\
$\mathrm{~S}_{\mathrm{IV}}$ & 143.2 & $3 \mathrm{~S}_{\mathrm{m}}=369$ & Pass & \\
\hline
\end{tabular}

Note: The local film stress intensity $\mathrm{S}_{\text {II }}$, the $\mathrm{S}_{\mathrm{IV}}$ shall be computed according to the operating load, the $\mathrm{S}_{\mathrm{m}}$ is the design stress intensity.

\section{Conclusion}

(1) The equivalent stress of the adsorption column mainly concentrates in the range of $0 \sim 104.84 \mathrm{MPa}$ at the internal pressure of $7.2 \mathrm{MPa}$ and the alternating temperature of $40 \sim 290^{\circ} \mathrm{C}$, which is less than the yield strength of the column. The points with abnormally large overall stress values all appear at the M2 opening chamfering of the tower body, and the maximum stress is $313.34 \mathrm{MPa}$.

(2) Under the action of temperature alternating load, the tower section $A \sim D$ all trigger stress concentration, the maximum stress is located in the inner chamfering of the pipe wall. The stress concentration of cross section C and D is the most obvious, and the stress concentration coefficient reaches 2.54 and 2.55 , respectively.

(3) The distribution of equivalent stress on the inner and outer surface of the joint position between the head and the shell and the joint position between the skirt and the shell has a large non-uniformity. The stress on the inner surface decreases first and then increases, and the stress on the outer surface increases first and then decreases. The maximum stress also appears in the area near the opening.

(4) The corresponding stress linearization results of each assessment path were evaluated and passed. The calculation results provide a theoretical basis for strength design and life prediction of adsorption tower under complex temperature alternation.

\section{Acknowledgement}

This work is supported by the key research and development projects of sichuan province (2020YFG0180), national natural science foundation of China (51974271), and Xinjiang oilfield company project of PetroChina (Safety risk analysis and countermeasure study of natural gas cryogenic temperature alternating condition).

\section{References}

1. I. Barsoum, S.A Lawal, R.J Simmons, et al. Failure analysis of a pressure vessel subjected to an internal blast LOAD $[\mathrm{J}]$. Engineering Failure Analysis, 2018, 91:354-369.

2. A. Lietzmann, J. Rudolph, E. Wei. Failure modes of pressure vessel components and their consideration in analyses[J]. Chemical Engineering \& Processing Process Intensification, 1996, 35(4):287-293.

3. Lee H.S, Yoon J.H, Park J.S, et al. A study on failure characteristic of spherical pressure vessel[J]. Journal of Materials Processing Technology, 2005, 164(none):882-888.

4. Chen xiezhong, Chemical equipment design book chemical container design[M], Shanghai: Shanghai Scientific \& Technical Publishers, 1987.12.

5. K. Hashimoto, K. Onizawa, R. Kurihara, et al. Thermal and stress analyses of the reactor pressure vessel lower head of the three-mile island unit 2[J]. International Journal of Pressure Vessels and Piping, 1992, 52(1): 25-40.

6. V. Chaudhry, A. Kumar, S.M. Ingole, et al. Thermomechanical Transient Analysis of Reactor Pressure Vessel[J]. Procedia Engineering, 2014, 86: 809-817.

7. D. Ferreño, R. Lacalle, I. Gorrochategui, et al. Analysis of dynamic conditions during thermal transient events for the structural assessment of a nuclear vessel[J]. Engineering Failure Analysis, 2009, 17(2010): 894-905.

8. A. Kandil. Analysis of thick-walled cylindrical pressure vessels under the effect of cyclic internal pressure and cyclic temperature[J]. International Journal of Mechanical Sciences, 1996, 38(12):13191332 .

9. Liu L, Li M.N, Ma X.Q. Structural stress and fatigue analysis of the molecular sieve absorption tower based on ANSYS[J]. Chemical Engineering \& Machinery, 2020, 47(03): 322-326.

10. Wang Y.R, Liu J, Tang J.P. Fatigue analysis of membrane LNG ship under alternating temperature load[J].Ship Engineering,2012,34(03):24-27.

11. Shen J, Liu X.Y. The Thermal Stress Analysis of Concrete Bridge Tower[J]. Applied Mechanics \& Materials, 2014, 638-640:1008-1011.

12. Song M.C. Estimation of instantaneous thermal stress on vessel wall or vessel noir [J]. Refining Equipment Design, 1982(03): 11-18.

13. Feng Z.R, Shen J, Wang X.J. Finite Element Analysis of Thermal Stress for Cable-Stayed Bridge Tower with Cracks[J]. Applied Mechanics and Materials, 2012, 178-181. 